\title{
Peces y sedimentos: dos matrices para la vigilancia de la contaminacion por sustancias peligrosas en la cuenca del rio Ebro.
}

\author{
C. Duran Lalaguna, A. Puig Infante, L. Pinilla Lopez-Oliva.
}

Area de Calidad de Aguas. Confederacion Hidrografica del Ebro. Po Sagasta, 24-28. 56071 Zaragoza. Tel.: 976 221993. FAX 976 214596. e-mail: cduran@chebro.es

\section{RESUMEN}

La Directiva 76/464/CEE obliga a los Estados Miembros a eliminar la contaminacion causada por sustancias peligrosas clasificadas como de Lista I, para ello preve la implantación de medidas de vigilancia en la fuente de emision y/o en el medio receptor. Basicamente el control en la fuente se realiza por imposición de un límite de emision en la autorizacion de vertido para cada una de las sustancias de lista I que se vierten a traves de las aguas residuales. En el medio receptor se debe asegurar y vigilar que se respeta el objetivo de calidad establecido para cada una de las sustancias en el agua, el sedimento y la biota. La Confederacion Hidrografica del Ebro ejecuta estas obligaciones limitando la emision de las sustancias en la autorizacion de vertido con las normas de emision legales. Además ha establecido la Red de Control de Sustancias Peligrosas cuya finalidad es vigilar el cumplimiento del objetivo de calidad de las sustancias de lista I en el agua, el sedimento y los peces susceptibles de estar contaminados, es decir, localizados en puntos aguas abajo de los focos de emision reales o posibles. La estandarizacion de la red finalizó en 1997; consta de 9 estaciones y se analiza: mercurio; cadmio; DDT y metabolitos; aldrin; endrin; dieldrin; isodrin; triclorobencenos; hexaclorobenceno; hexaclorobutadieno; $\mathrm{HCH}$ e isomeros; pentaclorofenol; cloroformo, tetracloruro de carbono; tricloroetileno, percloroetileno y 1,2-dicloroetano, las matrices que se controlan son agua, sedimento y peces. Con este articulo se pretende exponer los criterios aplicados en el diseño de la Red de Control de Sustancias Peligrosas con el fin de promover el intercambio de informacion sobre la mejor aplicación de la Directiva 76/464/CEE.

Palabras clave: agua, sedimento, biota, peces, sustancias organicas, compuestos organoclorados, metales, sustancias peligrosas, Directiva 76/464/CEE, objetivos de calidad, Lista I.

\begin{abstract}
According to European Directive 76/464/EEC, Member States must take appropriate action to eliminate pollution of waters by the dangerous substances included in List I. Two basic approaches will achieve this objective, i.e. an emission limit value (ELV) approach, and a water quality objective (WQO) based approach. The ELV approach requires that all discharges into waters including substances in List I need authorisation. Thus, emission standardsfor substances in List I must be laid down. The WQO-based approach ensures that the area affected by discharges complies with fixed quality objectivesfor water: sediments and biota. The Confederacion Hidrográfica del Ebro (CHE) has implemented the Directive by limiting all discharges including List I compounds. In discharge authorisations by CHE, emission limits are fixed. In addition, CHE has established a "DangerousSubstances Monitoring Network", to test the compliance of waters, sediments and biota with quality standards (i.e. the WQO-based aproach), downstream of every point source. The network was standardised in 1997. Today, there are nine sampling stations, and samples are analysedfor the following pollutants: mercury, cadmium; HCH and isomers; DDT and metabolites; carbon tetrachloride; pentachlorophenol; aldrin, dieldrin, endrin and isodrin; hexachlorobenzene; hexachlorobutadiene; chloroform; 1,2 - dichloroethane (EDC); trichloroethylene (TRI); perchloroethylene (PER); and trichlorobenzene $(T C B)$. These substances are monitored in water; sediments and biota. This article exposes the criteria applied to design the Dangerous Substances Monitoring Network, with an aim to informing about proper implementation of Directive 76/464/EC.
\end{abstract}

Key Words: water: sediment, biota, fish, organic substances, organochlorine compounds, metals, dangerous substances, 76/464/EEC Directive, Quality Objectives, List I. 


\section{INTRODUCCIÓN}

En el ambito de la UE se regula la protección de las aguas frente a la contaminacion causada por sustancias peligrosas a traves de un conjunto de directivas derivadas de la Directiva 76/464/CEE. En este marco, un compuesto quimico se considera peligroso si es toxico, persistente y bioacumulable que, en siglas, se denomina compuesto PBT. Por persistencia se entiende la capacidad de las sustancias de permanecer en el medio ambiente durante un período de tiempo largo, por bioacumulacion la tendencia a acumularse en los tejidos animales y por toxicidad la posibilidad de provocar efectos negativos en el ecosistema.

La directiva 761464 distingue dos categorias de sustancias peligrosas: las de lista I y las de lista II; los compuestos de lista I son especialmente peligrosos y por ello debe eliminarse la contaminacion que provocan. Dado que los de lista II son menos perjudiciales, la medida a aplicar es la reducción de la contaminacion para asegurar que su concentracion en el medio ambiente no cause efectos negativos. Las directivas promulgadas por la Comision para eliminar la contaminacion por sustancias de lista I fijan los limites de emision (en adelante LE) que se pueden autorizar en los vertidos de aguas residuales asi como los objetivos de calidad (en adelante OCA) que se deben respetar en el medio afectado por un vertido. Son sustancias de lista I todos aquellos compuestos sobre los que se ha legislado: mercurio; cadmio; DDT y metabolitos; aldrin; endrin; dieldrin; isodrin; triclorobencenos; hexaclorobenceno; hexaclorobutadieno; $\mathrm{HCH}$ e isomeros; pentaclorofenol; cloroformo, tetracloruro de carbono; tricloroetileno; percloroetileno y 1,2-dicloroetano. Por regla general, si se autoriza un vertido con los LE legales no es necesario vigilar el respeto del OCA pero, en la Confederación Hidrografica del Ebro (en adelante CHE) se ha decidido controlar ambos objetivos con el fin de asegurar una vigilancia mas integrada de la calidad de las aguas superficiales. Esta vigilancia se realiza a través de la Red de Control de Vertidos y de la Red de Control de Sustancias Peligrosas en Aguas Superficiales (en adelante RCSP) El OCA que debe respetarse en el agua es una concentracion concreta del orden de $1 \mathrm{mg} / \mathrm{l}$; sin embargo, en sedimentos y biota no es una concentracion específica sino un criterio que recibe el nombre de principio de standstill y que se fundamenta en que la concentracion de la sustancia en estas matrices, no aumente de forma significativa con el tiempo, por 10 tanto, deben vigilarse las tendencias temporales de los compuestos.

Este articulo pretende exponer los principios sobre los que se ha basado el diseño de la red que analiza el agua, sedimento y la biota (peces) en tramos afectados o susceptibles de ser afectados por vertidos de sustancias de lista I con el fin de vigilar el respeto del OCA. La directiva 761464 y derivadas son poco explicitas en los criterios que determinan las caracteristicas de la red, por 10 tanto se ha recurrido a un equipo interdisciplinar de expertos para su disefio. Esta red se inicio en 1992 y quedo definitivamente establecida en 1997 tras un trabajo de optimización y estandarizacion de la misma. Se compone de 9 estaciones (Tabla 1) distribuidas a lo largo de la Cuenca del Ebro ubicadas aguas abajo de vertidos que emiten sustancias de lista I (control de contaminacion puntual) asi como de ciudades que pudieran ser posibles focos de emision (control de contaminacion difusa). Los compuestos analizados son los menciados en el parrafo anterior. Las matrices que se controlan son agua, sedimento y peces. Dado que todos los Organismos de Cuenca deben establecer una red de estas caracteristicas, se considera interesante exponer los criterios de disefio ya que, si se unifican las variables que determinan la red, es mas facil comparar los resultados y asi facilitar el diagnóstico del estado de las aguas continentales. Actualmente, se ha ampliado la red a 18 puntos con el fin de valorar la contaminacion causada por sustancias de lista II.

\section{ESTACIONES DE MUESTREO}

En el disefio de una red es importante determinar además de las Estaciones de Muestreo, el punto exacto de toma de muestra ya que si no esta bien seleccionado puede invalidar todo el trabajo siguiente, aunque la analitica se realice respetan- 
Table 1. Estaciones de Control. Location of sampling stations.

\begin{tabular}{lll}
\hline \multicolumn{1}{c}{ CÓDIGO } & ESTACIÓN & VIGILANCTA \\
\hline ESTACIÓN T-1 & Rio Gállego en Jabarrella & Poligono Industrial de Sabiñánigo \\
\hline ESTACION T-2 & $\begin{array}{l}\text { Rio Ebro en Pina de Ebro } \\
\text { Poligonos de la periferia }\end{array}$ & Zaragoza ciudad \\
\hline ESTACION T-3 & Rio Ebro en Flix & Industria Quimica \\
\hline $\begin{array}{l}\text { ESTACION T-4 } \\
\text { Poligono industrial }\end{array}$ & Rio Segre en Torres de Segre & Lérida ciudad \\
\hline $\begin{array}{l}\text { ESTACION T-5 } \\
\text { Poligono de Barbastro }\end{array}$ & Rio Cinca en Monzon & Poligono de Monzon \\
\hline $\begin{array}{l}\text { ESTACION T-6 } \\
\text { Poligonos industriales }\end{array}$ & Rio Arga en Ororbia & Pamplona ciudad \\
\hline ESTACION T-7 & Rio Ebro en Miranda de Ebro & Industrias Quimicas de Lantaron \\
\hline $\begin{array}{l}\text { ESTACION T-8 } \\
\text { Poligonos industriales }\end{array}$ & Rio Zadorra en Trespuentes & Vitoria ciudad \\
\hline $\begin{array}{l}\text { ESTACION T-9 } \\
\text { Poligono industrial }\end{array}$ & Rio Ebro en Campredó & Tortosa ciudad \\
\hline
\end{tabular}

do las normas de calidad mas exigentes. La directiva seiiala como criterio para ubicar el punto de toma 10 siguiente:

"las muestras deberan tomarse en un punto 10 suficientemente cercano al vertido para que puedan ser representativas de la calidad del medio acuatico en la region afectada por los vertidos" (ref. punto 7, Sección B, Anexo I Directiva 86/280/CEE).

Es decir, unicamente establece el criterio de representatividad del punto, y en el diseño de la red se ha interpretado como que la estacion de muestreo debe localizarse aguas abajo de un vertido industrial, fuera de la camara de mezcla y, a la vez, 10 suficientemente cercano al foco de emision de manera que esté directamente influenciado por el mismo.

Consideramos además que debe garantizarse la repetividad del muestreo es decir que la toma se realice exactamente en el mismo punto de campaiia en campaiia, y asi calcular la tendencia tem- poral de los contaminantes en el medio afectado, esta medida es especialmente significativa cuando se valora el sedimento.

Ademas, para cada matriz se consideran los siguientes criterios especificos:

Agua: la toma debe efectuarse en el mismo lugar que la de peces.

Sedimento: la toma debe realizarse en un punto donde se asegure la existencia de sedimento de año en año, por 10 tanto, no se recomienda seleccionar puntos en zonas cercanas a presas o zonas afectadas por avenidas. Ademas la textura del sedimento debe contener una proporción importante de fracción limosa-arcillosa que es donde preferentemente se acumulan las sustancias contaminantes.

Peces: la toma se realiza mediante pesca elk trica lo que condiciona el punto ya que debe ser posible el uso de esta tecnica. En principio se intenta que la pesca se realice 10 mas cerca posible de la toma del sedimento. 


\section{FRECUENCIA DE MUESTREO}

La directiva 76/464 indica que la frecuencia de muestreo "debera ser suficiente para poner en evidencia las eventuales modificaciones del medio acuatico, teniendo en cuenta, en particular, las variaciones naturales del regimen hidráulico" (ref. punto 7, Sección B, Anexo I de la Directiva 86/280/CEE). La aplicacion de este criterio no es igual en el agua, en el sedimento o en los peces y se ha establecido de la siguiente manera:

Agua: se considera que la frecuencia minima que permite valorar los cambios estacionales es de 2 veces al aiio, una en periodo de caudal medio y la otra en caudal de estiaje.

Sedimento: se fija como frecuencia suficiente un control anual. Se recomienda que la toma se realice en periodos de bajo caudal por razones de seguridad de los operarios y accesibilidad al terreno. La frecuencia propuesta se apoya con la bibliografia; Rice et al, (1993) defienden que no existen diferencias estacionales significativas en la concentracion de contaminantes organicos PCBs, DDTs, -PAHs- en tres zonas diferentes de California. Tate et al (1996) tambien toman muestras de peces y de sedimentos una sola vez al aiio en un estudio realizado para valorar la concentracion de compuestos organoclorados en sedimento superficial y peces del South Platte River Basin entre Colorado, Nebraska y Wyoming, USA. Por su parte, Sola y Canton (1990) estudiando la contaminación por metales pesados en sedimentos superficiales de los rios de Guipuzcoa ofrecen, una media de los datos recogidos en dos meses diferentes del mismo aiio.

Peces: se establece como frecuencia optima la anual, preferentemente entre los meses de septiembre y octubre ya que durante el resto del año es menos recomendable por las siguientcs razones: en mayo y junio no es aconsejable porque la mayoria de las especies se encuentran en pleno periodo de reproducción. En los meses anteriores a la freza, las hembras suelen aumentar de peso para garantizar el éxito reproductivo, de modo que, aumenta considerablemente el contenido en grasa corporal que es donde mas se acumulan los plaguicidas. Si la toma fuera en esas fechas los resultados finales podrian alterar las conclusiones. Raldua et al (1997) encuentran fuertes variaciones en el contenido de HCHs y DDTs en barbos capturados en primavera y en verano. Además, al finalizar el periodo reproductivo que suele ser en verano, los individuos pierden hasta el $25 \%$ de su peso corporal por 10 que se alterarian los resultados. Cuando comienza el invierno, algunas especies hibernan y tampoco es aconsejable la pesca. Para finalizar, a partir de mediados de octubre aumenta la probabilidad de avenidas en los cauces por 10 que la toma de muestras se hace poco accesible y peligrosa.

\section{MATRICES ANALIZADAS}

La legislacion obliga a respetar los OCA en tres tipos de matrices: agua, sedimento y biota que puede ser moluscos y/o crustaceos y/o peces. En la estandarizacion de la red se ha tomado como matriz significativa el pez sin analizar las muestras de los otros organismos. El OCA en aguas suele ser un valor de concentracion concreto, del orden de $1 \mathrm{mg} / 1$ y en la matrices sólidas se debe asegurar el respeto del "principio de standstill", es decir, que la concentracion no aumente de forma significativa con el tiempo. De los resultados de control se concluye que desde 1992, aiio en el que se inicio la RCSP, nunca se han superado los valores estipulados para el agua. La evaluacion de los resultados en sedimentos y peces es mas dificil ya que la valoracion del OCA requiere una cantidad de datos 10 suficientemente grande como para que se pueda someter a tratamientos estadisticos que permitan analizar las tendencias temporales de las sustancias en estas matrices. Dado que la estandarizacion de la Red se realizó en 1998, no es posible todavia realizar dicho estudio estadistico. Un análisis de los resultados del control de forma individual ha servido para prestar especial atencion a alguna de las estaciones en las que se han detectado valores puntuales altos en algunos ejemplares de peces. Concretamente en la estacion que controla el rio Cinca en Monzon, se observaron valores de p,p'DDE por encima de los encontrados en la biblio- 
grafia consultada y por ello, se ha realizado un estudio mas profundo de la zona para localizar el origen de esta concentración.

La RCSP analiza las siguientes matrices:

Agua: se analizan muestras puntuales

Sedimento: en esta matriz debe fijarse la fraccion granulometrica que hay que analizar y respecto a cual se expresan los resultados (incluido si es muestra seca o humeda) de lo contrario, los resultados nunca seran comparables entre sí. En la literatura consultada existe una gran variedad de propuestas sobre el tamaño idoneo del grano de sedimento y se extiende desde menor de $\mathbf{6 3}$ $\mathrm{mm}$ hasta $2000 \mathrm{~mm}$. En la RCSP de la CHE se ha establecido que se analice la fraccion inferior a $200 \mathrm{~mm}$ y que el resultado se exprese respecto a esta fraccion eliminada la humedad.

Peces: dado que el objetivo de la red es vigilar las tendencias temporales (principio de standstill), cuando se analizan peces es necesario fijar un mayor numero de variables. En la CHE se ha considerado como elementos esenciales a definir en cada estacion:

Las especies seleccionadas en cada tramo;

La talla (o edad) de los individuos que refleja el tiempo de exposicion;

El numero de individuos que integran una muestra y

La matriz u organo a analizar.

En la tabla 2 se recogen las especies seleccionadas en cada estacion y la talla de los individuos capturados. Siempre que se pueda se intenta seleccionar una especie representativa de fondo y otra depredadora. Esta estandarizacion se ha realizado segun los estudios que se discuten a continuación:

\section{Especies seleccionadas}

En la Directiva 76/464 se indica que "la autoridad competente elegira las especies de peces que haya de considerar como indicadores para el análisis". Solo para el cadmio se especifica que, si es posible, se analice Mytilus edulis.

La selección de especies se puede hacer siguiendo varios criterios:
Table 2. Relación de Especies Piscícolas Seleccionadas en cada Estacion. Selected fish species for analyses in each sampling station.

\begin{tabular}{|c|c|c|}
\hline ESTACIÓN & ESPECIE & TALLA $(\mathrm{cm})$ \\
\hline \multirow[t]{2}{*}{$\mathrm{T}-1$} & Madrilla & $10-20$ \\
\hline & Barbo & $25-45$ \\
\hline \multirow[t]{2}{*}{$\mathrm{T}-2$} & Madrilla & $10-20$ \\
\hline & Carpa & $25-45$ \\
\hline \multirow[t]{2}{*}{$\mathrm{T}-3$} & Alburno & $10-15$ \\
\hline & Carpa & $25-45$ \\
\hline \multirow[t]{2}{*}{$\mathrm{T}-4$} & Carpa & $25-45$ \\
\hline & Alburno & $10-15$ \\
\hline \multirow[t]{2}{*}{$\mathrm{T}-5$} & Barbo & $25-45$ \\
\hline & Alburno & $10-15$ \\
\hline \multirow[t]{2}{*}{$\mathrm{T}-6$} & Carpa & $25-45$ \\
\hline & Carpin & $12-26$ \\
\hline \multirow[t]{2}{*}{$\mathrm{T}-7$} & Carpin & $12-26$ \\
\hline & Barbo & $25-45$ \\
\hline \multirow[t]{2}{*}{$\mathrm{T}-8$} & Barbo & $25-45$ \\
\hline & Bermejuela & $12-20$ \\
\hline \multirow[t]{2}{*}{$\mathrm{T}-9$} & Carpa & $25-45$ \\
\hline & Escardinio & $12-20$ \\
\hline
\end{tabular}

1" Deben analizarse las especies bioindicadoras de los parametros a medir. Este criterio es dificil de aplicar ya que se trata de medir 25 sustancias y no existen de momento especies indicadoras de tantos compuestos.

2" Deben analizarse las especies mas abundantes en la zona.

3" Deben analizarse las especies de captura más facil.

En la CHE se han aplicado los criterios 2 y 3 y las orientaciones encontradas en bibliografia para elegir un buen indicador biologico:

. Facil de identificar taxonomicamente.

. Facil de recolectar.

. Distribución cosmopolita.

.Estar asociadoa abundantes datos autoecologicos. . Interes economico, nutritivo o intrinseco

. Rapidez para acumular sustancias presentes en el medio externo. 
. Facilmente cultivables en laboratorio.

. Presentar baja variabilidad genética.

Además, se han seguido las pautas del National Contaminant Biomonitoring Program (en adelante NCBP) que es el Programa desarrollado por el Gobiemo de Estados Unidos durante más de 20 aiios en el que midieron metales y sustancias organocloradas en peces de 112 estaciones situadas en los ríos mas grandes de Estados Unidos y en los grandes lagos. Las muestras analizadas eran compuestas de 3-5 animales y se analizaba el pez completo. En cada Estacion se capturaba una especie depredadora y otra de fondo (Schmitt et al., 1990)

En principio, si es posible, en cada punto se ha seleccionado dos especies indicadoras, una de fondo y la otra depredadora, basandose en clasificacion segun los tipos de alimentacion de Garcia de Jalon et al., (1993)

\section{Talla de los individuos capturados}

Para comparar resultados de aiio en año, se recomienda que se aplique un criterio que de continuidad a los resultados. En la mayoria de los estudios publicados suele ser la edad del individuo, que se puede determinar contando las líneas de crecimiento en los otolitos o en las escamas aunque ambos métodos son susceptibles de error. Para solventar este problema, se recurre a determinar la edad por medio de la talla. Se seleccionan individuos de la misma talla en cada especie y en las sucesivas pescas se trata de buscar de ese tamaño. Este criterio es el seguido por el programa NCBP ya citado. Las tallas seleccionadas para cada especie representada son las de su talla comun.

\section{Numero de muestras}

La Directiva 76/464/CEE tambien especifica que "deberan realizarse los analisis sobre un numero suficiente representativo de muestras y de especies" En la RCSP se toman al menos tres individuos de cada especie con el mismo tamaiio y peso que constituyen una sola muestra denominada pool.

\section{Matriz}

Para seleccionar la matriz se pueden aplicar dos criterios distintos:

. Utilizar visceras: higado, riñón, cerebro, etc. ya que la mayoria de plaguicidas y metales pesados tienen un organo diana que es donde se acumulan preferentemente.

. Utilizar el pez entero. La ventaja de utilizar el pez entero en este estudio de biomonitorizacion es que se evita el error generado por una posible movilización de sustancias debida al estres al que se somete al animal asi como la probabilidad de encontrar un individuo con la viscera diana daiiada.

En la RCSP de la CHE se ha adoptado como matriz de analisis el pez entero.

\section{MÉTODOS DE TOMA DE MUESTRAS}

En la Directiva $76 / 464$ no se referencia ni se aconseja ningun metodo de muestreo. En la RCSP de la CHE se procede de la siguiente manera:

Agua: las muestras se toman de forma manual, siguiendo el protocolo de trabajo desarrollado por el Laboratorio de Calidad de las Aguas de la CHE.

Sedimento: el metodo habitual es la draga tipo Van Veen que se lanza desde un puente, barca, presa, etc. Si no es posible utilizar draga se toma desde la orilla manualmente con rascadores de fondo.

Peces: con carácter general y si las caracteristicas del rio lo permiten, se capturan con pesca electrica, tal como se recomienda en bibliografia (Garcia de Jalon et al., 1993) porque es menos selectiva que la pesca con caña, el pez sufre 10 menos posible y se obtiene una muestra representativa de la zona muestreada. Todos los trabajos consultados (e.g. Schmitt and. Brumbaugh, 1990; Chevreuil et al.) utilizan equipos de pesca electrica que ocasionalmente complementan con trasmallos. Desafortunadamente el uso de la pesca eléctrica tiene algunas limitaciones; por ejemplo el rio no puede ser muy profundo, como maximo hasta la cintura del muestreador, además si lleva 
mucha corriente cabe el peligro de las caidas. Dado que hay que transportar el equipo de pesca hasta la orilla del rio es necesario que exista facil accesibilidad. Por ultimo la reacción de los peces ante la descarga electrica no es exactamente igual, los oseos conducen mejor la electricidad que los cartilaginosos, además afecta mas a los peces de mayor longitud, cuando el animal esta enfermo, agotado o con productos sexuales maduros no reacciona bien.

\section{TRANSPORTE Y PREPARACIÓN DE LAS MUESTRAS}

Agua: las muestras destinadas al analisis de metales se acondicionan con acido a $\mathrm{pH}<2$ y las de sustancias organicas se transportan sin la adicion de ningún estabilizante. Si los compuestos que se van a analizar son sustancias volatiles se emplean frascos sellados evitando la formacion de la camara de aire.

Sedimento: las muestras para el analisis de metales se recogen en bolsas de polietileno mientras que las destinadas al control de organoclorados se transportan envueltas en dobles hojas de papel de aluminio. Se dejan secar a temperatura ambiente o en estufa y después se pasan por cribas para recoger el sedimento inferior a $200 \mathrm{~mm}$. El método que se sigue es el de pasta saturada y humedad de saturation del U.S. Salinity Laboratory Staff (1969).

Peces: los peces se congelan hasta el momento del analisis. De cada especie se hace un pool de todo el pez con al menos 3 ejemplares de la misma talla aunque se recomienda que utilicen 10 individuos. Cadapool se divide en dos, uno para el analisis de sustancias organicas que se conserva en papel de aluminio y el otro para metales que se conserva en bolsas de polietileno.

\section{SUSTANCIAS ANALIZADAS Y MÉTODOS ANALÍTICOS}

Actualmente, los metales y compuestos organicos que se analizan en la RCSP son todos los de Lista I. Durante este año se ha previsto incorporar las sustancias preferentes de lista II sobre las que España ha definido OCA en el RD 99512000.

En la tabla 3 se han recopilado los métodos de analisis que se aplican.

Las sustancias de Lista I analizadas son:

. Metales: Mercurio y Cadmio

. Sustancias Organicas Volatiles: Cloroformo; tetracloruro de carbono; 1,2-dicloroetano; tricloroetileno y percloroetileno

Sustancias Organicas Semivolatiles: a-HCH; b-HCH; d-HCH; c-HCH; p,p'-DDT; o,p'-DDT;

Table 3. Metodologia Analitica. Analytical techniques.

\begin{tabular}{lll}
\hline MATRIZ & CONTAMINANTE & MÉTODO ANALÍTICO \\
\hline AGUA & Cadmio & EPA 200.8 sobre muestra filtrada \\
& Mercurio & ISO 5666-1:1983 \\
& Organica Volatil & EPA 624 \\
& EPA 525 \\
\hline \multirow{2}{*}{ Organica Semivolatil } & EPA 200.8 y extraccion EPA 3051 \\
& Cadmio & IS0 5666-1:1983 y extraccion EPA 3051 \\
& Mercurio & No se analiza \\
& Organica Volatil & GC/MS previa extraccion con soxhlet \\
\hline \multirow{2}{*}{ Organica Semivolatil } & Cadmio & EPA 200.8 $\mathbf{y}$ extraccion EPA 305 1 \\
& Mercurio & IS 0 5666-1:1983 y extraccion EPA 3051 \\
& Organica Volatil & Go se analiza \\
& Organica Semivolatil & GC/MS previa extraccion con soxhlet \\
\hline
\end{tabular}


p,p'-DDE; p,p'-DDD; hexaclorobenceno; hexaclorobutadieno; pentaclorofenol; aldrin, endrin, isodrin, dieldrin y los tres triclorobencenos

Las sustancias organicas volatiles no se analizan ni en el sedimento ni en el pez.

\section{BIBLIOGRAFÍA}

CHEVREUIL M., A. CARRU, A. CHESTERIKOFF, P. BOET, E. TALES \& J. ALLARDI. 1995. Contamination of fish from different areas of the river Seine (France) by organic (PCB and pesticides) and metallic ( $\mathrm{Cd}, \mathrm{Cr}, \mathrm{Cu}, \mathrm{Fe}, \mathrm{Mn}, \mathrm{Pb}$ and $\mathrm{Zn})$ micropollutants. The Science of the Total Environment, 162: 31-42

GARCÍA DE JALÓN D., M. MAYO, F. HERVELLA, E. BARCELO \& T. FERNANDEZ. 1993. Principios y técnicas de gestidn de la pesca en aguas continentales. Ed. Mundi Prensa. Madrid. 247 pp.

RALDÚA D., P. FERRANDO, C. DURÁN, \& C. PEDROCCHI. 1997. The Influence of Place of Capture, Sex, and Season on the Organochlorine Pesticide Content in Barbel (Barbus Graellsi) from Northeastern Spain. Chemosphere, 35: 2245-2254
RICE, C. P. R., B. SELTENRICH, M. L. SPIES \& M.L. KELLER. 1993. Seasonal and annual distribution of organic contaminants in marine sediments from Elkhorn Slough, Moss Landing Harbor and Neasrshore Monterey Bay, California. Environ. Pollut., 82 : 79-91.

SCHMITT C. J. \& W. G. BRUMBAUGH. 1990. National Contaminant Biomonitoring Program: Concentrations of Arsenic, Cadmium, Copper, Lead, Mercury, Selenium, and Zinc in U.S. Freshwater Fish, 1976-1984. Arch. Environ. Con. Tox. 19: 731-747

SOLA M. J. y L. CANTON. 1990. Contaminación por metales pesados en sedimentos superficiales de los rios de Guipuzcoa. Lurralde, 13: 165-172

TATE C. M. \& J. S. HEINY. 1996. Organoclhorine Compounds in Bed Sediment and Fish Tissue in the South Platte River Basin, USA, 1992- 1993. Arch. Environ. Con. Tox. 30: 62-78

U.S. SALINITY LABORATORY STAFF. 1969. Saline and Alkali Soils. USDA Handbook nº6, 160 $\mathrm{pp}$

UNIÓN EUROPEA. 1976. Directiva del Consejo 76/464. DOCE L $129 \mathrm{del} 18.5 .1976 .23 \mathrm{pp}$.

UNION EUROPEA. 1980. Directiva del Consejo 86/280 DOCE L 181 de 4.6.86. 16 pp. 